\title{
Print and online versions of Evidence-Based Nursing: innovative teaching tools for nurse educators
}

The availability of Evidence-Based Nursing $(E B N)$ online to institutions that currently subscribe to the print version is good news for nurse educators. $E B N$ online offers educators an innovative way to use the journal's resources and the internet to teach students about evidence-based practice (EBP). Each issue of $E B N$ online contains the full text (downloadable and printable) of all abstracts of clinical studies, commentaries, and editorials that are in the print version. Although the print version of $E B N$ is certainly a useful tool to help nurse educators enhance student learning, the advantage of an online version of the journal is that it contains direct links to the original published research report that is summarised in the abstract, and to many of the references cited in the commentary. Thus, when students are given assignments using $E B N$ online, they learn about a fast and efficient internet source of high quality clinical research.

If nurse educators want their students to value and appreciate clinical research, they must help them to see that accessing and keeping up with the latest findings are neither difficult nor burdensome. Synopses journals such as EBN are a good evidencebased resource for busy practitioners and for busy students who are just learning about how research findings relate to clinical practice. Medical school faculties are beginning to use synopses journals in their teaching. Guyatt et al discuss the effectiveness of this approach: "In our residency programme we have observed that even trainees who are less interested in evidence-based methods develop a respect for, and ability to track down and use, secondary sources of pre-appraised evidence (evidencebased resources) that provide immediately applicable conclusions." ${ }^{1}$ This same approach holds promise for nursing students. In the following sections, examples are provided to show how nurse teachers can use the print and online versions of $E B N$ to augment and enhance the content of both classroom based and clinically based nursing courses.

\section{Using EBN in classroom based courses}

$E B N$ can be used in nursing foundation courses to introduce students to pre-appraised clinical evidence early in their nursing studies and to show them how textbook information can be enhanced by current clinical research findings. When students learn about the nursing process, they can read relevant synopses or abstracts from EBN. For example, an EBN abstract describes a study that showed that telephone assessment and teaching by nurses improved glycaemic control in clinic patients with diabetes. ${ }^{2}$ Just 6 minutes of nurse telephone contact each month, in addition to automated assessment and self care education phone calls, resulted in improved patient outcomes. These patients had fewer problems with medication adherence, fewer diabetic symptoms, and a lower mean serum glucose concentration than similar patients who did not receive this intervention. The accompanying commentary stressed the exciting possibilities the technology offers for enhancing nursing assessment and patient education.

Another abstract describes a systematic review of nursing interventions for smoking cessation. ${ }^{3}$ This abstract could be used to provide students with a positive example of how nursing interventions can affect unhealthy patient behaviours. The accompanying commentary discusses other important considerations, such as the need for nurses to time interventions to coincide with patient readiness and the prudence of eliciting support from patients' partners or family members. The online version of this $E B N$ abstract contains a link to a clinical practice guideline on smoking cessation. This acquaints students with a guideline that includes practical, empirically based recommendations to assist nurses and other healthcare providers in their efforts to help patients stop smoking.

The concept of advanced directives or "living wills" is a common topic of introductory nursing courses in the US, and several $E B N$ abstracts summarise studies of the effectiveness of advanced directive programmes and seminars. One abstract summarises the findings of a study that showed that an advanced directive programme in nursing homes resulted in fewer hospital admissions and hospital days and lower hospital and healthcare costs than control nursing homes without such programmes. ${ }^{4}$ The accompanying commentary describes findings from other related studies, and the online version provides links to the abstracts of these studies in Medline.

Another abstract describes a study that found that a brief group seminar with written documents was more effective than mailed information in increasing the number of advanced directives among medical outpatients ${ }^{5}$. The accompanying commentary includes suggestions for the types of healthcare settings where the study findings might be most relevant and the online version provides a link to the abstract of a related study in Medline. All of this information would enrich classroom discussion and enhance students' learning about advanced directives.

Using $E B N$ abstracts and commentaries and the links on the online version to augment student learning in nursing foundation courses provides the dual benefit of stimulating interesting classroom discussion and promoting research utilisation behaviours that may later become habitual.

$E B N$ content can also be useful in more advanced nurse education. For example, $E B N$ includes several abstracts of studies focusing on elements of cardiovascular nursing. One such summary concerns a systematic review of the effectiveness of psychoeducational programmes in reducing the incidence of recurrent myocardial infarction, cardiac risk factors, and cardiac mortality. ${ }^{6}$ Cardiac patients who attended psychoeducational programmes had better outcomes than similar patients who did not participate in such programmes. The commentary contrasts these findings with those from 2 other reviews on the same topic, helping students to gain a better perspective of the overall state of research in this area. $E B N$ online provides a link to the full text of one of these reviews, which gives students immediate access to detailed information.

Several abstracts of studies examining the care and treatment of children with asthma could be assigned as readings for students taking paediatric nursing courses. One abstract presents the findings of a study that examined the effectiveness of a clinical pathway for children admitted to hospital with 
asthma exacerbations. ${ }^{7}$ Findings showed that when a clinical pathway was used to guide care, children needed less frequent treatment with nebulised $B$ agonists and had shorter hospital stays than similar children who did not receive care guided by the pathway. Similar findings from other studies are discussed in the commentary, with a direct link to the full text report of one of these studies.

If the assigned reading for nursing courses regularly included abstracts and commentaries from $E B N$, the habit of using research evidence is more likely to become established in students before they graduate and enter into nursing practice.

\section{Using EBN in clinically based courses}

$E B N$ is an excellent resource for teaching students about research findings that enhance their understanding of clinical experiences. Nurse teachers could have students use $E B N$ to identify studies of relevance to patients in their clinical area. Each week, students could pick one abstract and commentary to discuss during a clinical tutorial. These discussions would allow everyone in the clinical group to learn new evidence-based information on a regular basis. Nurse teachers could help students to identify the nursing implications of study findings and consider how they might use the findings in their practice. One example is an abstract and commentary about a large cohort study that found that the risk of fracture markedly increased immediately after a stroke. ${ }^{8}$ Another abstract and commentary present the findings of a qualitative study of patient perceptions of stroke rehabilitation. ${ }^{9}$ Both studies have important implications for students and other clinicians who care for stroke patients. Using research findings to enhance students' understanding of their clinical experiences helps to solidify their commitment to evidence-based practice.

\section{Using $E B N$ in a nursing research course}

Finally, $E B N$ publishes a great deal of material that would be useful in nursing research courses. The abstracts themselves provide clear examples of focused clinical questions, the use of various research designs, and appropriate samples; they describe interventions, outcomes, and statistical findings that are meaningful to practitioners and their patients. The commentaries can help students to identify the nursing implications of study findings and begin thinking about research utilisation in practice. The online version links with other citations, including many full text articles, to offer students greater detail about specific research studies. Additionally, the editorials (particularly the EBN Notebooks and Users' Guides) describe and explain various research concepts, including how to find and select valid research for application in practice. These editorials use papers abstracted in $E B N$ as illustrative examples.

\section{Summary and conclusion}

The movement towards evidence-based practice encourages practitioners to use research evidence to guide their clinical decisions and challenges nurse educators to cultivate, in their students, the habit of using research evidence. To accomplish these goals, educators must go beyond teaching research in a formal course or assigning research articles as reading material. New tools and teaching strategies are required to help students develop the habit of regularly using research to guide practice. Because nursing practice in current times can be energy draining and time constraining, students need to learn ways of quickly and efficiently locating and digesting current research findings, and they need guidance to comprehend the implications of research findings for nursing practice. $E B N$ is a practical and effective tool that can help meet these needs. The online version offers users the advantage of being searchable across all issues of the journal. Additionally, readers who subscribe to $E B N$ can use the email alert system on the web version to keep apprised of new research in their specific areas of interest. In summary, both the print and online versions of $E B N$ offer many exciting possibilities for nurse educators to enhance student learning using current clinical research.

MARSHA FONTEYN, RN, PhD
University of San Francisco San Francisco, California, USA

1 Guyatt GH, Meade MO, Jaeschke RZ, et al. Practitioners of evidence based care. Not all clinicians need to appraise evidence from scratch but all need some skills. BMJ 2000; 320:954-5.

2 Automated telephone assessment and education with nurse follow up improved self care and glycaemic control in patients with diabetes [abstract]. Evidence-Based Nursing 2000;3:117. Abstract of: Piette JD, Weinberger M, McPhee SJ, et al. Do automated calls with nurse follow-up improve self-care and glycemic control among vulnerable patients with diabetes? Am J Med 2000;108:20-7.

3 Review: nursing interventions increase smoking cessation rates in adults. Evidence-Based Nursing 2000;3:47. Abstract of: Rice VH, Stead LF. Nursing interventions for smoking cessation. (Cochrane Review, latest version 21 May 1999). In: Cochrane Library. Oxford: Update Software.

4 An advance directive programme in nursing homes reduced health services use without affecting patient satisfaction. Evidence-Based Nursing 2000;3:125. Abstract of: Molloy DW, Guyatt GH, Russo R, et al. Systematic implementation of an advance directive program in nursing homes: a randomized controlled trial. JAMA 2000;283:1437-44.

5 Seminars on advance directives for adult outpatients increased completion rates. Evidence-Based Nursing 1998;1:53. Abstract of: Landry FJ, Kroenke K, Lucas C, et al. Increasing the use of advance directives in medical outpatients.J Gen Intern Med 1997;12:412-15.

6 Review: psychoeducational programmes reduce long term mortality and recurrence of myocardial infarction in cardiac patients. Evidence-Based Nursing 2000;3:80. Abstract of: Dusseldorp E, van Elderen T, Maes S, et al. A meta-analysis of psychoeducational programs for coronary heart disease patients. Health Psychol 1999;18:506-19.

7 A clinical pathway reduced use of nebulised $B$ agonists and length of hospital stay in children with asthma exacerbations. Evidence-Based Nursing 2001;4:44. Abstract of: Johnson KB, Blaisdell CJ, Walker A, et al. Effectiveness of a clinical pathway for inpatient asthma management. Pediatrics 2000; 106:1006-12.

8 Fracture risk was increased after stroke with a marked increase immediately after stroke. Evidence-Based Nursing 2001;4:122. Abstract of: Kanis J, Oden A, Johnell O. Acute and long-term increase in fracture risk after hospitalization for stroke. Stroke 2001;32:702-6

9 Patients in stroke units have better outcomes, but receive less personal nursing care. Evidence-Based Nursing 2001;4:128. Abstract of: Pound P, Ebrahim S. Rhetoric and reality in stroke patient care. Soc Sci Med 2000;51:1437-46 